\title{
A New Optimal Sensor Placement Strategy Based on Modified Modal Assurance Criterion and Improved Adaptive Genetic Algorithm for Structural Health Monitoring
}

\author{
Can He, ${ }^{1}$ Jianchun Xing, ${ }^{1}$ Juelong Li, ${ }^{2}$ Qiliang Yang, ${ }^{1}$ Ronghao Wang, ${ }^{1}$ and Xun Zhang ${ }^{1}$ \\ ${ }^{1}$ College of Defense Engineering, PLA University of Science and Technology, Nanjing 210007, China \\ ${ }^{2}$ Technical Management Office of Naval Defense Engineering, Beijing 100841, China \\ Correspondence should be addressed to Can He; hecan19880828@163.com
}

Received 7 July 2014; Accepted 23 August 2014

Academic Editor: Zheng-Guang Wu

Copyright (C) 2015 Can He et al. This is an open access article distributed under the Creative Commons Attribution License, which permits unrestricted use, distribution, and reproduction in any medium, provided the original work is properly cited.

Optimal sensor placement (OSP) is an important part in the structural health monitoring. Due to the ability of ensuring the linear independence of the tested modal vectors, the minimum modal assurance criterion (minMAC) is considered as an effective method and is used widely. However, some defects are present in this method, such as the low modal energy and the long computation time. A new OSP method named IAGA-MMAC is presented in this study to settle the issue. First, a modified modal assurance criterion (MMAC) is proposed to improve the modal energy of the selected locations. Then, an improved adaptive genetic algorithm (IAGA), which uses the root mean square of off-diagonal elements in the MMAC matrix as the fitness function, is proposed to enhance computation efficiency. A case study of sensor placement on a numerically simulated wharf structure is provided to verify the effectiveness of the IAGA-MMAC strategy, and two different methods are used as contrast experiments. A comparison of these strategies shows that the optimal results obtained by the IAGA-MMAC method have a high modal strain energy, a quick computational speed, and small off-diagonal elements in the MMAC matrix.

\section{Introduction}

With the development of constructive technology, more and more large-scale structures, such as suspension bridges, television towers, sea wharfs, and high-rise buildings, have been built all over the world. However, due to harsher environment, these civil infrastructures may be damaged by strong wind force, torrential rain, severe earthquake, explosion, and other abnormal loads [1]. The traditional routine visual inspection usually ignores the tiny failures and the flaws inside the structures. Therefore, structural health monitoring (SHM) [2-4] has become an important research topic in the engineering protection field. By monitoring the force condition of the structures real-timely, SHM system can detect the anomalies in response and find the possible deterioration at early stage to ensure the safety of the structures. A typical SHM system usually includes three subsystems: sensor subsystem, data processing subsystem, and condition evaluation subsystem [5]. Sensor subsystem is a fundamental part of SHM system. Modal parameter identification, damage detection, and structural condition evaluation are all based on the data acquired from the sensors. Generally, the more sensors are placed, the more information could be obtained. Nevertheless, the number of sensors is strictly constrained by the high cost of purchase and maintenance for the sensors. For instance, there are only 23, 65, and 72 accelerometers instrumented in the Tsing Ma Bridge, Tingkau Bridge, and Jiangyin Bridge, respectively [6]. The sensors number is far less than the available positions. Therefore, how to deploy the limited sensors becomes a challenging task.

The traditional expert experience method is effective for the simple structures. Nevertheless, for a large-scale structure that has a large volume and complex geometry, it is very difficult to determine the optimal sensor locations only by experience. In the past few years, many technologies have been developed to achieve optimal sensor placement (OSP), such as modal kinetic energy method [7,8], effective independence method [9, 10], QR decomposition method [11, 12], and 
information entropy method [13, 14]. Carne and Dohrmann [15] considered that distinguishing one modal vector from another is essential to realize modal parameter identification and proposed a famous OSP method named minMAC. The minMAC method includes two parts. The first section is modal assurance criterion (MAC). In the criterion, the offdiagonal element of the MAC matrix is considered to be an index for evaluating the angle of the corresponding two modal vectors. The smaller element indicates bigger angle and less correlation between two modal vectors. The second section is to achieve sensor placement using MAC. First, some locations are selected as an initial set based on experience and structural topology, and the remaining locations are considered as a candidate set. Second, the candidate locations are added one by one to the initial set and the combination that minimizes the maximum off-diagonal element of the MAC matrix is selected as the new initial set. Third, the second step is repeated till the fixed sensors number is gained.

Although minMAC is considered as an effective OSP method and used widely, some defects still exist. Firstly, the core idea of MAC is to evaluate the correlation of the modal vectors for different placement scheme, but the modal energy of the selected location cannot be guaranteed. It means that some degree of freedoms (DOFs) with low signal to noise ratio may be selected as the optimal sensor positions; this defect could decrease the precision of the acquired data and the accuracy of modal parameters identification. Secondly, the minMAC method has a large computational complexity. To determine one location, the minMAC method needs to compute the MAC matrices of the variable combinations until all candidate locations have been selected. In order to improve the computational efficiency of the minMAC method, computational intelligence technology has been used, such as simulated annealing algorithm [16], ant colony optimization algorithm [17], particle swarm optimization algorithm [18], and monkey algorithm [19]. Due to easy coding method and quick evolution velocity, genetic algorithm (GA) has become a hot research direction. Liu et al. [20] presented a modified GA using two-dimensional array coding method instead of binary coding method; generalized genetic algorithm (GGA) was proposed by Yi et al. [21] to solve the OSP problem for high-rise buildings. Javadi et al. [22] adopted a hybrid intelligent method which was based on a combination of neural network and GA. Although these modifications make great progress, there are still some defects existing in GA [23]. The main drawback is that the crossover and mutation factors are invariant during the whole iteration cycle; this defect drops the searching ability of GA.

The objective of this study is to present a modified minMAC method that has a big modal energy index and a quick computational speed. A new method termed IAGAMMAC is proposed to achieve this goal. First, a modified modal assurance criterion (MMAC) is proposed to improve the modal energy of the selected locations by constructing the new modal shape matrix. And then, an improved adaptive genetic algorithm (IAGA), which uses root mean square of off-diagonal elements in the MMAC matrix as the fitness function, is adopted to determine the optimal sensor locations. The excellent calculation efficiency and global optimization ability of IAGA assist to enhance the computational speed of IAGA-MMAC.

The remaining parts of the paper are organized as follows. Section 2 introduces the traditional MAC and presents MMAC. The IAGA-MMAC strategy is described in Section 3. Section 4 provides a case study of sensor placement on a numerically simulated wharf structure to verify the effectiveness of the IAGA-MMAC method. Section 5 is the conclusion and the future work.

\section{The Modified Modal Assurance Criterion}

2.1. Introduction of the Traditional MAC. A basic requirement to distinguish the measured modes is that the measured modal vectors must be as linearly independent as possible. MAC provides a useful criterion to evaluate the correlation of modal vectors. MAC matrix is defined as

$$
\operatorname{MAC}_{i j}=\frac{\left(\Phi_{i}^{T} \Phi_{j}\right)^{2}}{\left(\Phi_{i}^{T} \Phi_{i}\right)\left(\Phi_{j}^{T} \Phi_{j}\right)}
$$

where $\Phi_{i}$ and $\Phi_{j}$ are the $i$ th and $j$ th column vectors in the modal shape matrix $\Phi$.

The off-diagonal elements in the MAC matrix express the correlation between two modal vectors. Identifying the $i$ th mode and the $j$ th mode is easy if the value of $\mathrm{MAC}_{i j}$ is small.

2.2. Description of MMAC. The main idea of MAC is to guarantee the linear independence of the modal vectors. However, some DOFs with low energy may be selected as sensor locations using MAC; this defect could decrease the signal to noise ratio of the acquired data and make troubles in modal shape identification and structural damage detection.

As shown in (1), the element of the MAC matrix is computed using the column vectors of the modal shape matrix $\Phi$. In general, modal shape matrix should be composed by all orders of modes. But for the sake of reducing computation complexity, only a few modal orders are selected to compose the modal shape matrix. To improve the modal energy of the selected locations, the modal orders having large dynamical response should be selected. Therefore, how to evaluate the dynamical feature of different modal orders becomes an important work. In tradition, the first several modes are considered to have large dynamical response. Nevertheless, the modal energy of a structure is not always concentrated on the low-order modes. To some complex structures, such as high-rise building and suspension bridge, some high modal orders also have large dynamical response [24]. Therefore, it is not suitable that only the first several modes are selected to compose the modal shape matrix. In order to improve the modal energy of sensor locations, a modified MAC is presented to settle the issue in this study. In the new criterion, modal participation factor (MPF) is presented as the criterion to evaluate the dynamical response of different modal orders. And then, the new modal shape matrix $\bar{\Phi}$ is composed by the modal orders with big MPF. Finally, the modified MAC matrix is computed using $\bar{\Phi}$. 
2.2.1. Modal Order Selection Using MPF. Different choices of modal order result in the various placement results. According to the requirement of OSP, the modal order having a strong dynamic response should be selected. Nevertheless, modal order is selected based on experience in the traditional methods. In order to solve this problem, MPF is presented as the evaluation criterion for the modal order selection.

The equation of motion for the $n$ DOF dynamic system is represented as

$$
M \ddot{u}+C \dot{u}+K u=-M D \ddot{u}_{g},
$$

where $M$ is the structural mass matrix, $C$ is the structural damping matrix, $K$ is the structural stiffness matrix, $D$ is the vector describing the excitation direction, $u$ is the modal displacement vector, and $\ddot{u}_{g}$ is the acceleration generated by input force.

The displacement vector $u$ can be transformed by

$$
u=\Phi \cdot q
$$

where $\Phi$ is the modal shape matrix and $q$ is the modal coordinate.

If $M, C$, and $K$ meet the orthogonalization of the normalization modal shape, (2) can be written as

$$
\ddot{q}_{i}+2 \zeta_{i} w_{i} \dot{q}_{i}+w_{i}^{2} q_{i}=-\frac{\Phi_{i}^{T} M D}{\Phi_{i}{ }^{T} M \Phi_{i}} \ddot{u}_{g}(t),
$$

where $q_{i}$ is the $i$ th modal coordinate, $w_{i}$ is the natural frequency of the $i$ th mode, and $\zeta_{i}$ is the fraction of critical damping of the $i$ th mode.

The MPF is defined as follows in [25]

$$
\varphi_{i r}=\frac{\Phi_{i}^{T} M D_{r}}{\Phi_{i}^{T} M \Phi_{i}}
$$

where $\varphi_{i r}$ is the MPF of the $i$ th mode in $r$ direction.

The mass normalization for the modal shape matrix $\Phi$ is determined by

$$
\Phi_{i}^{T} M \Phi_{i}=1 .
$$

Based on (5) and (6), the MPF can be expressed as

$$
\varphi_{i r}=\Phi_{i}^{T} M D_{r}
$$

where $D_{r}$ is a vector describing the excitation in $r$ direction, and it can be computed as

$$
D_{r}^{T}=T \cdot E_{r}
$$

where $E_{r}$ is a unit vector in $r$ direction and $T$ is a constant matrix that indicates the relationship between the excitation and reference points:

$$
T=\left[\begin{array}{cccccc}
1 & 0 & 0 & 0 & Z-Z_{0} & Y_{0}-Y \\
0 & 1 & 0 & Z_{0}-Z & 0 & X-X_{0} \\
0 & 0 & 1 & Y-Y_{0} & X_{0}-X & 0 \\
0 & 0 & 0 & 1 & 0 & 0 \\
0 & 0 & 0 & 0 & 1 & 0 \\
0 & 0 & 0 & 0 & 0 & 1
\end{array}\right],
$$

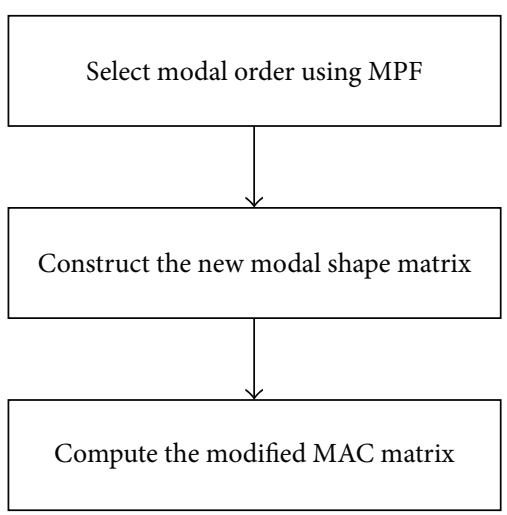

Figure 1: Computation process of the modified MAC matrix.

where $X_{0}, Y_{0}$, and $Z_{0}$ are the global Cartesian coordinates of the reference point and $X, Y$, and $Z$ are the global Cartesian coordinates of the excitation point.

MPF reflects the amplitude of dynamical response for all the modes. Therefore, the modal order that has big MPF should be selected priorly to construct the new modal shape.

Although all of the modes can be sorted based on their MPFs, there is still a question that how many modes should be selected. The modal participating mass ratio is presented as a criterion to determine the number of the modes. Modal participating mass is defined as

$$
M_{p i}=\frac{\left(\Phi_{i}^{T} M D\right)^{2}}{\Phi_{i}^{T} M \Phi_{i}}
$$

where $M_{p i}$ is the modal participating mass of the $i$ th mode.

When mass normalization has been conducted for the modal shape matrix $\Phi,(10)$ can be transformed by

$$
M_{p i}=\left(\Phi_{i}^{T} M D\right)^{2}
$$

The modal participating mass ratio of the $i$ th mode can be expressed as

$$
r_{i}=\frac{M_{p i}}{D^{T} M D} .
$$

Wilson [25] suggested that the summation of modal participating mass ratio of the selected modes should be over $90 \%$. This criterion is employed in this study to determine the number of modes.

2.2.2. Computation of the MMAC Matrix. The dynamical response of different modal orders can be sorted by comparing their MPFs; the new modal shape matrix $\bar{\Phi}$ is constructed by the modal orders with big MPFs. Therefore, the modified MAC (MMAC) matrix can be computed as

$$
\operatorname{MMAC}_{i j}=\frac{\left(\bar{\Phi}_{i}^{T} \bar{\Phi}_{j}\right)^{2}}{\left(\bar{\Phi}_{i}^{T} \bar{\Phi}_{i}\right)\left(\bar{\Phi}_{j}^{T} \bar{\Phi}_{j}\right)}
$$

The computation process of the modified MAC matrix is shown as Figure 1. 


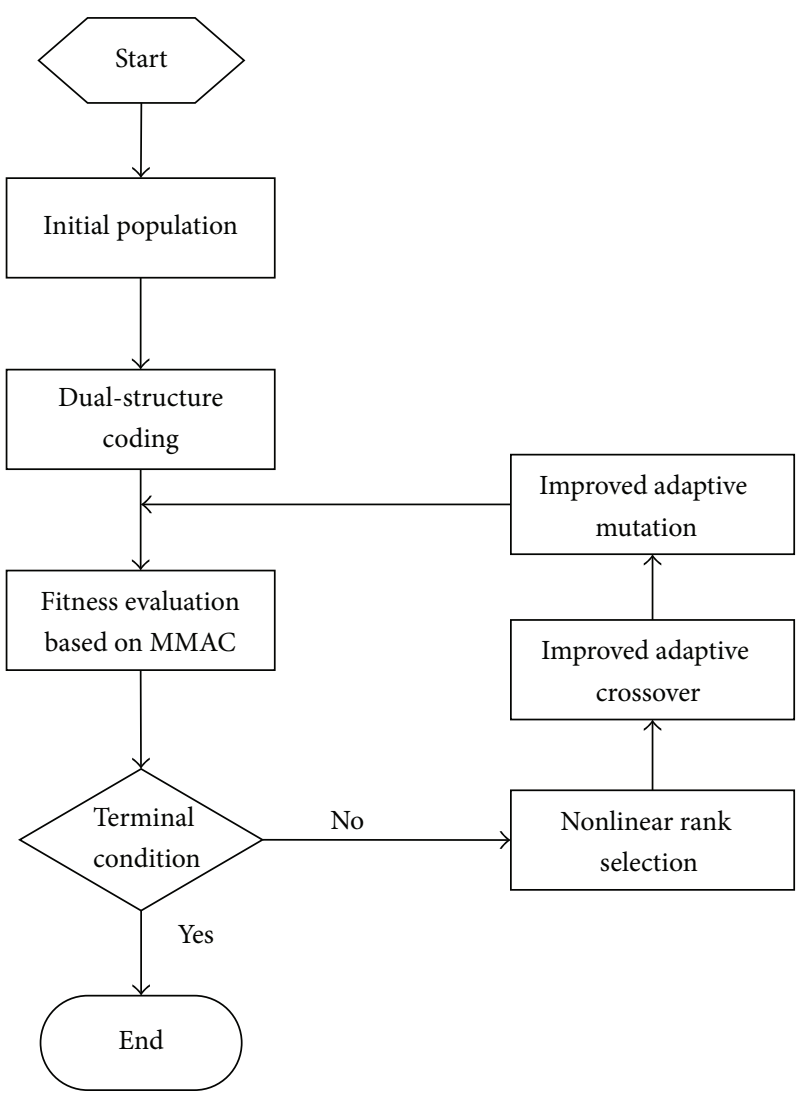

FIGURE 2: Flowchart of the IAGA-MMAC method.

\section{Optimal Sensor Placement Based on the IAGA-MMAC Method}

The traditional GA has a shortcoming that its crossover and mutation factors are stationary during the whole iteration cycle; this defect could drop the evolution velocity and searching ability of the algorithm. Therefore, a new OSP method named IAGA-MMAC is presented to settle the issue. The flowchart of the IAGA-MMAC is shown in Figure 2.

3.1. Coding Method. In the traditional coding method, the gene number of value 1 could be changed in the processes of crossover and mutation, which may result in two or more sensors placed on one location. Therefore, the dual-structure coding method is used in this study. The chromosome of individual is composed of two rows, where the upper row $s(i)$ named append code indicates all of the locations, and the lower row $X s(i)$ named variable code represents whether the location $s(i)$ places a sensor with the value 0 or 1 . The dualstructure coding method is shown as Table 1.

3.2. Selection Scheme. The nonlinear ranking selection scheme is used to choose the good individual in one generation. Firstly, the individuals are sorted by comparing their fitness values. And then, the nonlinear function (14) is adopted to determine the selection probability of different individuals. Compared with the common roulette wheel selection
TABLE 1: Dual-structure coding method.

\begin{tabular}{lcclc}
\hline Location code & $s(1)$ & $s(2)$ & $\cdots$ & $s(n)$ \\
\hline Variable code & $X s(1)$ & $X s(2)$ & $\cdots$ & $X s(n)$ \\
\hline
\end{tabular}

scheme, this method does not depend on the concrete values of individual fitness; therefore, it can avoid the scale conversion of fitness values. This advantage can inhibit the algorithm premature convergence:

$$
P\left(x_{n}\right)=q(1-q)^{i-1},
$$

where $q$ is the selection probability of best individual and $i$ is the sort number of the fitness value of $x_{n}$.

3.3. Crossover and Mutation. The crossover and mutation factors can make great influence on the computation efficiency of GA. In the traditional GA, the factors keep invariant during the whole iteration cycle. This defect drops the evolution velocity and optimization ability of the algorithm. Therefore, the adaptive mechanism is introduced into this algorithm [26]. The adaptive adjustment processes of the two factors are defined as

$$
\begin{gathered}
P_{c}= \begin{cases}k_{1} \frac{\left(f_{c}-f_{\mathrm{avg}}\right)}{f_{\max }-f_{\mathrm{avg}}}, & f_{c} \geq f_{\mathrm{avg}}, \\
k_{2}, & f_{c}<f_{\mathrm{avg}},\end{cases} \\
P_{m}= \begin{cases}k_{3} \frac{\left(f_{m}-f_{\mathrm{avg}}\right)}{f_{\max }-f_{\mathrm{avg}}}, & f_{m} \geq f_{\mathrm{avg}}, \\
k_{4}, & f_{m}<f_{\mathrm{avg}},\end{cases}
\end{gathered}
$$

where $f_{\max }$ is the maximum of fitness value, $f_{\text {avg }}$ is the average of population fitness value, $f_{c}$ represents the bigger fitness value between the two crossover individuals, $f_{m}$ is the biggest fitness value among the mutation individuals, $k_{1}$ and $k_{2}$ are the adjustment coefficients of crossover factor, and $k_{3}$ and $k_{4}$ are the adjustment coefficients of mutation factor.

As shown in (15), when $f_{c}$ and $f_{m}$ are close or equal to $f_{\text {max }}$, the $P_{c}$ and $P_{m}$ will decline to zero. This problem leads to the good individuals update very slowly at the beginning of the evolution process. In order to address this issue, the improved adaptive crossover and mutation factors are presented as

$$
\begin{gathered}
P_{c}= \begin{cases}p_{1}-\frac{\left(p_{1}-p_{2}\right)\left(f_{c}-f_{\mathrm{avg}}\right)}{f_{\mathrm{max}}-f_{\mathrm{avg}}}, & f_{c} \geq f_{\mathrm{avg}}, \\
p_{1}, & f_{c}<f_{\mathrm{avg}},\end{cases} \\
P_{m}= \begin{cases}p_{3}-\frac{\left(p_{3}-p_{4}\right)\left(f_{m}-f_{\mathrm{avg}}\right)}{f_{\max }-f_{\mathrm{avg}}}, & f_{m} \geq f_{\mathrm{avg}}, \\
p_{3}, & f_{m}<f_{\mathrm{avg}},\end{cases}
\end{gathered}
$$

where $p_{1}$ and $p_{2}$ represent the maximum and minimum values of the crossover probability, respectively, and $p_{3}$ and $p_{4}$ are the maximum and minimum values of the mutation probability, respectively. 


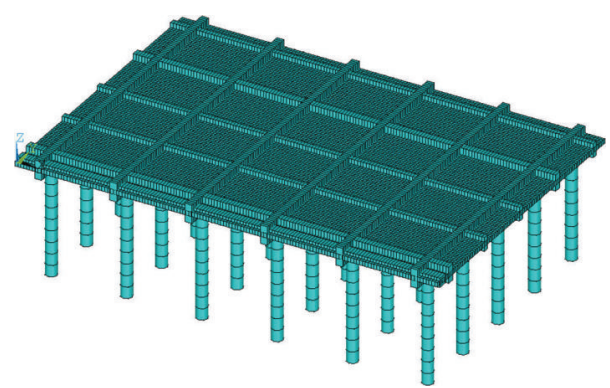

Figure 3: FE model of the wharf.

When $f_{c}$ and $f_{m}$ are close to $f_{\max }$, the new adaptive mechanism ensures that the crossover and mutation factors of good individual increased to $p_{2}$ and $p_{4}$. This advantage can help the good individual update quickly in the whole evolution cycle.

3.4. Fitness Function. The off-diagonal element of the MMAC matrix expresses the correlation between the corresponding two modal vectors. The elements are valued between 0 and 1 , and it is easy to identify the $i$ th mode and $j$ th mode if the

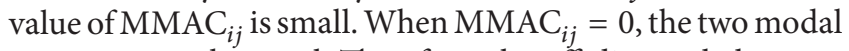
vectors are orthogonal. Therefore, the off-diagonal elements of MMAC matrix can be considered as an optimization objective for the OSP problem: the smaller element value represents the better sensor placement result.

The fitness function in IAGA is asked to be a maximization problem. Therefore, the fitness function is constructed as

$$
f=1-\sqrt{\frac{1}{N} \sum_{i=1}^{N} X_{i}^{2}}
$$

where $X_{i}$ is the off-diagonal elements of the MMAC matrix.

\section{Demonstration Case}

In order to verify the effectiveness of the IAGA-MMAC method, a case study of sensor placement on a numerically simulated wharf structure is provided below.

4.1. Modeling and Modal Analysis. A high-piled wharf is selected as a case study. The total length of wharf is $55 \mathrm{~m}$, and the width of anterior bearing platform is $30 \mathrm{~m}$. The upper structure of the platform is composition of cross beams, longitudinal beams, and reinforced concrete decks. The platform is supported by six rows of vertical piles, each row has five piles, and the distance between adjacent piles is $5.50 \mathrm{~m}$. The finite element (FE) model of the wharf is established using commercial software Ansys, and the FE model is shown in Figure 3.

The cross beams and longitudinal beams are modeled by beam 4 , vertical piles are simulated by pipe16, and reinforced concrete decks are built by shell63. 15147 nodes are generated in total. The natural frequency and modal shape of the
TABLE 2: Natural frequency of the first 10 modes.

\begin{tabular}{lccccc}
\hline Modal order & 1 & 2 & 3 & 4 & 5 \\
\hline Frequency $(\mathrm{Hz})$ & 2.592 & 2.620 & 2.818 & 6.902 & 7.339 \\
Mode order & 6 & 7 & 8 & 9 & 10 \\
Frequency $(\mathrm{Hz})$ & 7.501 & 8.011 & 8.261 & 9.035 & 9.948 \\
\hline
\end{tabular}

TABLE 3: Parameter setting.

\begin{tabular}{lccccc}
\hline Population & Generation & $P_{1}$ & $P_{2}$ & $P_{3}$ & $P_{4}$ \\
\hline 100 & 500 & 0.9 & 0.6 & 0.1 & 0.01 \\
\hline
\end{tabular}

structure are received as Table 2 and Figure 4 by modal analysis.

4.2. Computation of the MMAC Matrix. The MPFs of the modes are computed using (7) to (9). The results of the first 50 modes are shown in Figures 5, 6, and 7.

As shown in Figures 5 to 7 , the 1st, 3rd, 5th, and 11th modal orders have big MPFs in $x$ direction. The 2nd, 4 th, and 6th modal orders have large MPFs in $y$ direction. MPFs of the 4th, 6th, and 8th modal orders are big in $z$ direction. Therefore the 1st, 2nd, 3rd, 4th, 5th, 6th, 8th, and 11th modes are selected as the optimal combination. The modal participating mass ratio of the combination in each direction is computed using (10) to (12), and the results are presented as

$$
r_{x}=95.2 \% ; \quad r_{y}=95.1 \% ; \quad r_{z}=98.9 \% \text {, }
$$

where $r_{x}$ is the modal participating mass ratio in $x$ direction.

The results show that $r_{x}, r_{y}$, and $r_{z}$ are all over $90 \%$. Therefore, the selected modal order combination is reasonable.

The new modal shape matrix $\bar{\Phi}$ is composed as

$$
\bar{\Phi}=\left[\Phi_{1}, \Phi_{2}, \Phi_{3}, \Phi_{4}, \Phi_{5}, \Phi_{6}, \Phi_{8}, \Phi_{11}\right],
$$

where $\Phi_{i}$ is the $i$ th column vector of the original modal shape matrix $\Phi$.

The MMAC matrix is computed using $\bar{\Phi}$ according to (13).

4.3. Optimal Sensor Placement Using IAGA-MMAC. IAGA, which uses the off-diagonal elements of the MMAC matrix as fitness function, is adopted to achieve the sensor placement. According to the advice of [26], the parameters of IAGA are set as in Table 3.

The optimal process of IAGA-MMAC is executed according to Figure 2. The algorithm has been run for 50 times with a certain sensor number. The best fitness function values with different sensors numbers are shown in Figure 8.

As shown in Figure 8, when the sensor number is set between 5 and 11, the fitness increases with sensor added. The fitness function reaches its maximum when the sensor number is set at 11. But if the sensor number is more than 11, the fitness does not increase with sensor added. The reason for the contradiction is that the row vector determined at the newly included sensor location has a strong linear relationship with 


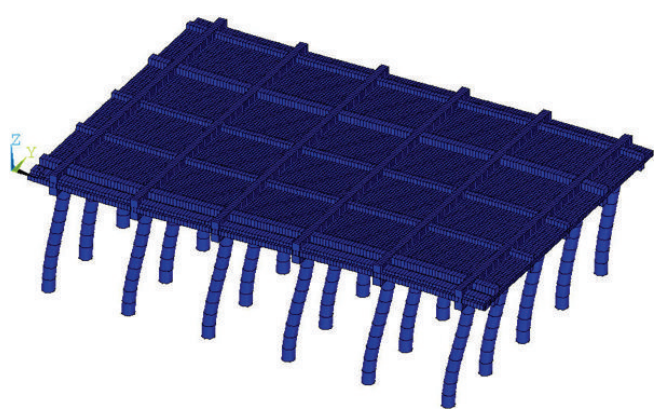

(a) 1st mode

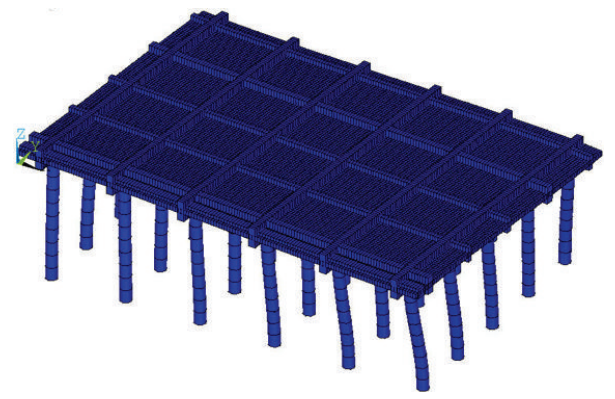

(c) 3rd mode

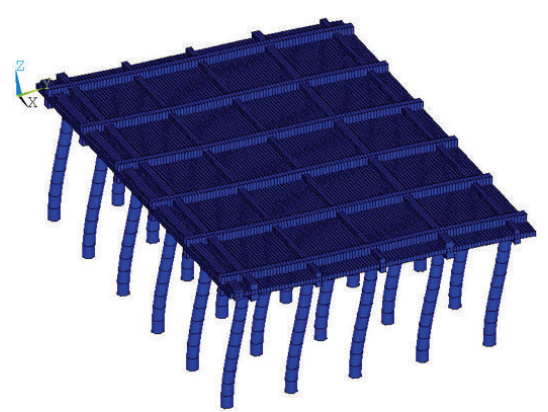

(b) 2nd mode

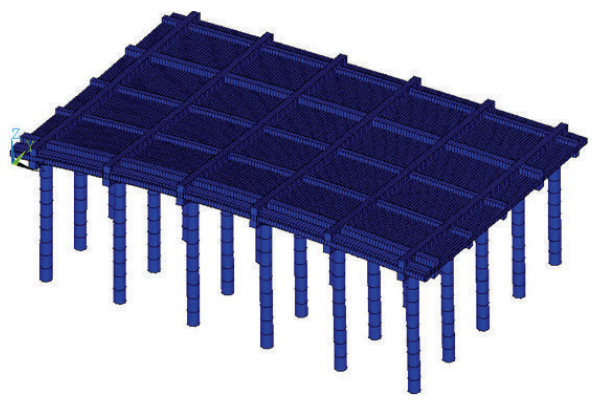

(d) 4th mode

FIgURE 4: The 1st-4th modes shape.

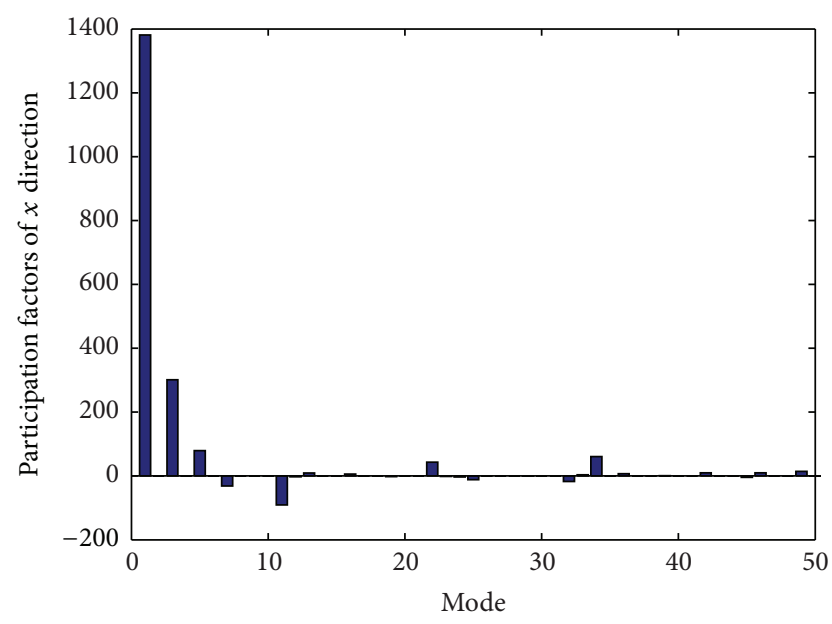

FIGURE 5: MPF of $x$ direction.

the entire previous sensor set. Therefore, eleven is determined as the optimal sensor number in consideration of placement cost and efficiency. The final optimal result is in Table 4.

4.4. Contrast Experiment. Three different methods are used to deploy the sensors for the wharf, including AGA with the traditional MAC as fitness function (AGA-MAC) [27], IAGA with the traditional MAC (IAGA-MAC), and IAGA-MMAC. The parameters of AGA are the same as IAGA. The optimal placement results of three methods are provided in Figures 9 , 10 , and 11.

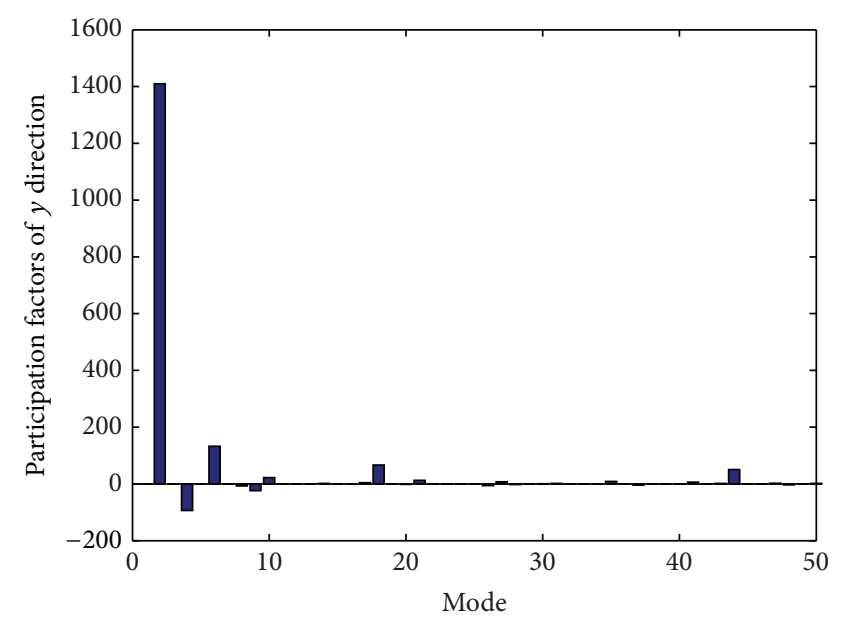

FIGURE 6: MPF of $y$ direction.

TABLE 4: Optimal sensor locations.

\begin{tabular}{lcccccc}
\hline Sensor number & 1 & 2 & 3 & 4 & 5 & 6 \\
\hline Node & 387 & 597 & 1445 & 2544 & 2769 & 3384 \\
Direction & $x$ & $z$ & $y$ & $z$ & $x$ & $z$ \\
Sensor number & 7 & 8 & 9 & 10 & 11 & \\
Node & 4272 & 5423 & 7392 & 11060 & 14028 & \\
Direction & $z$ & $x$ & $z$ & $y$ & $z$ & \\
\hline
\end{tabular}

The modal energy index and MAC index are selected to evaluate the placement effect of three methods. The summation of modal strain energy (MSE) for all the selected 


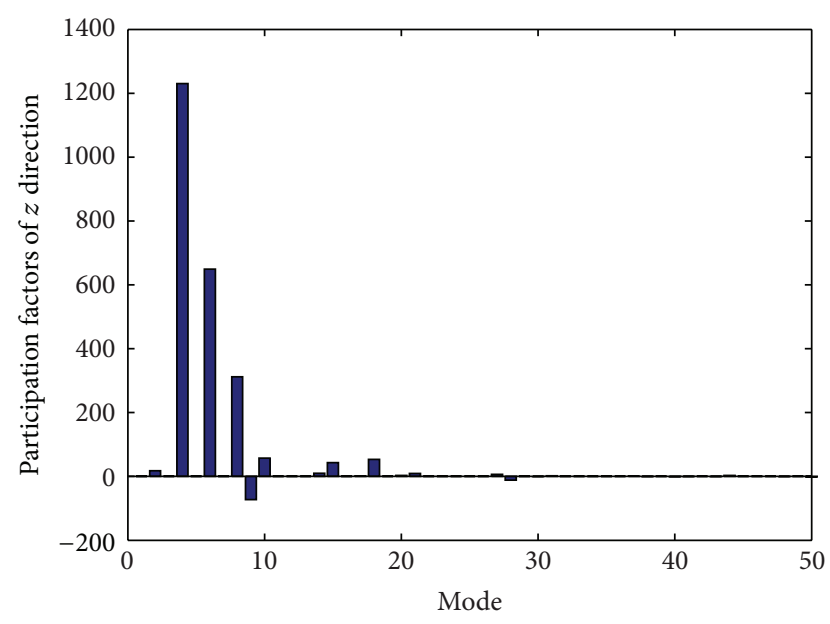

Figure 7: MPF of $z$ direction.

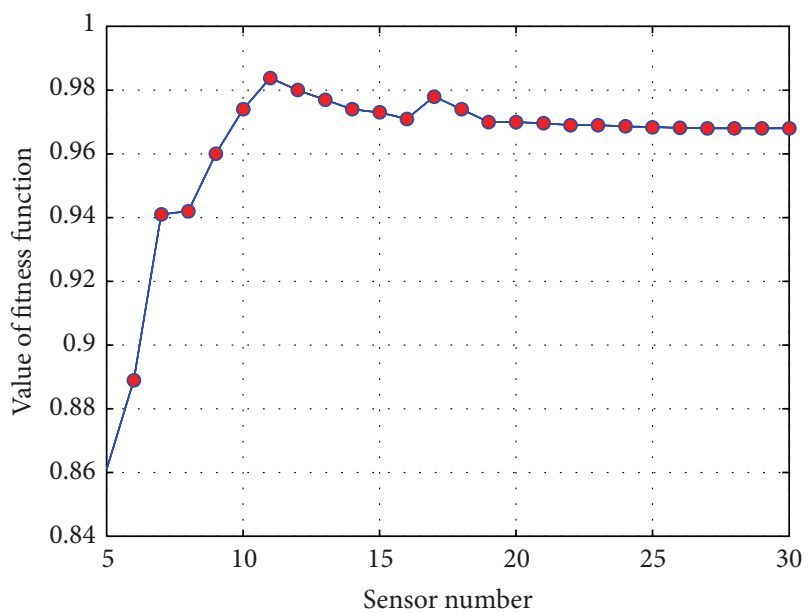

FIGURE 8: Fitness function values with different sensor numbers.

TABLE 5: MAC index of three methods.

\begin{tabular}{lcc}
\hline Method & $\begin{array}{c}\text { RMS of off-diagonal } \\
\text { elements }\end{array}$ & $\begin{array}{c}\text { Maximum of } \\
\text { off-diagonal elements }\end{array}$ \\
\hline AGA-MAC & 0.0349 & 0.1228 \\
IAGA-MAC & 0.0202 & 0.0631 \\
IAGA-MMAC & 0.0162 & 0.0371 \\
\hline
\end{tabular}

locations is shown in Figure 12, and the energy of single sensor location is provided by Figure 13.

The MAC matrices of three methods are shown in Figures 14, 15, and 16. The RMS and maximum of off-diagonal elements in the MAC matrix are provided in Table 5.

The evolution processes of three methods are shown in Figure 17.

4.5. Result Analysis. As shown in Figures 12 and 13, the MSE summation of all the selected locations by IAGA-MMAC is bigger than that by AGA-MAC and IAGA-MAC obviously, and the MSE of single sensor location by IAGA-MMAC is also much larger. These results imply that IAGA-MMAC is

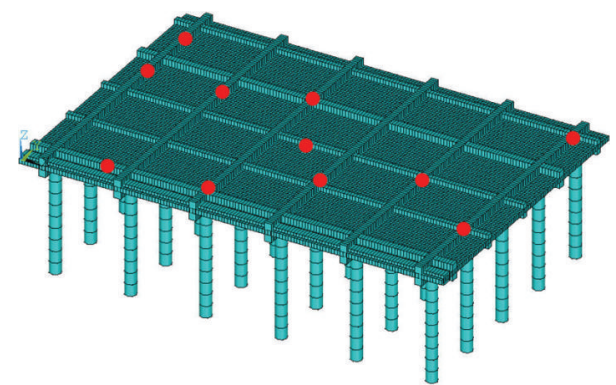

Figure 9: Sensor placement result of AGA-MAC.

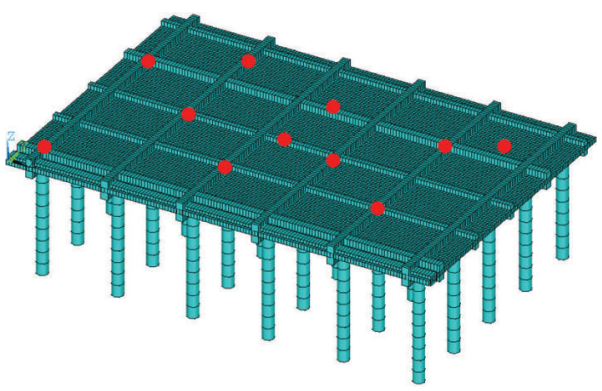

FIGURE 10: Sensor placement result of IAGA-MAC.

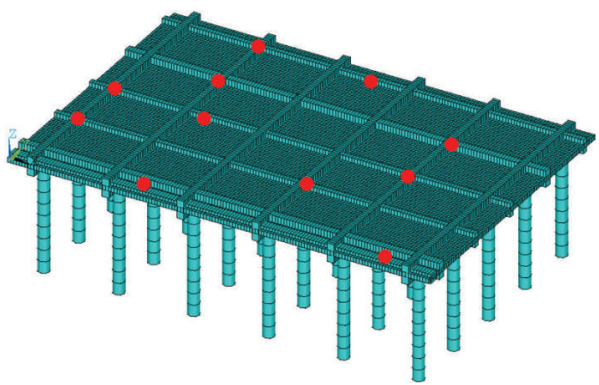

FIgURE 11: Sensor placement result of IAGA-MMAC.

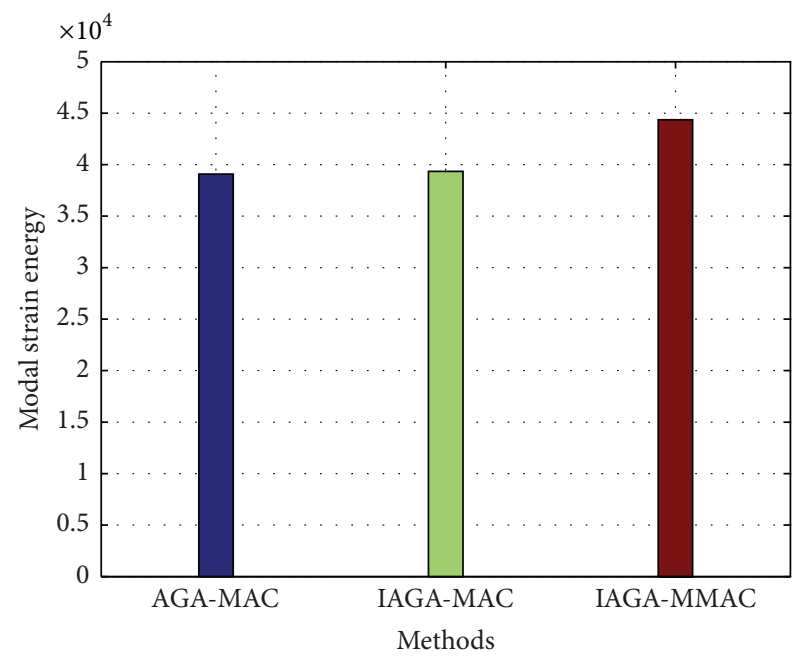

FIGURE 12: MSE summation of all the sensor locations. 


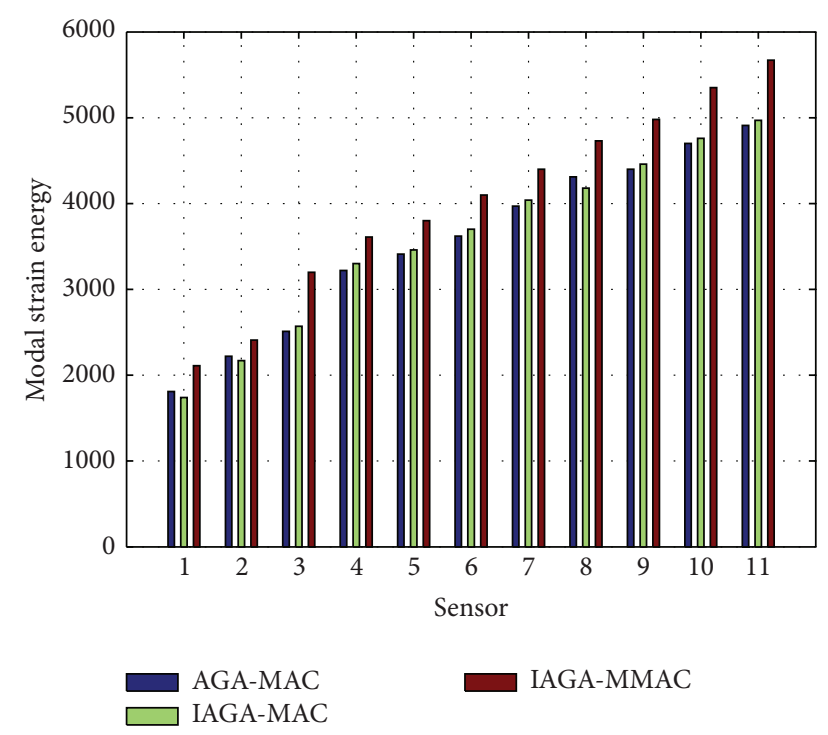

Figure 13: MSE of single sensor location.

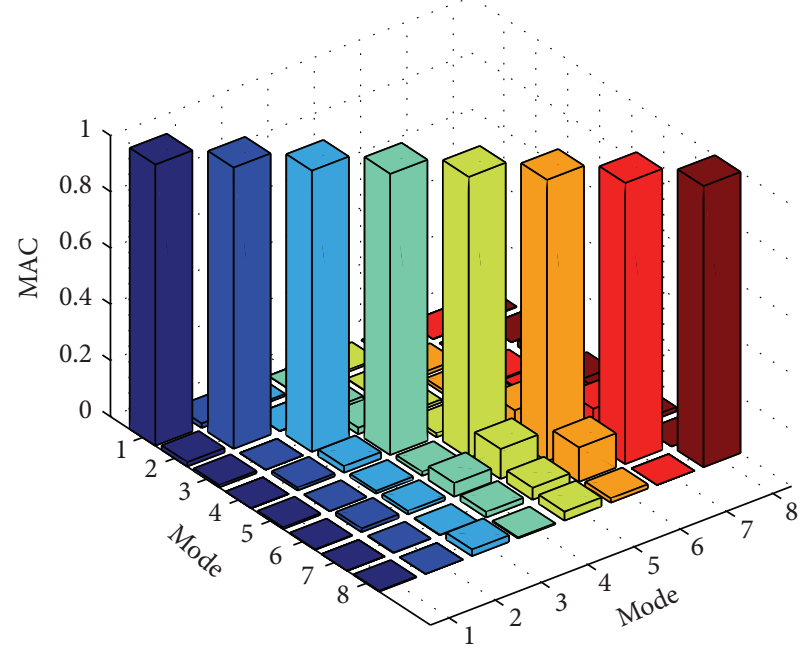

Figure 14: MAC matrix of AGA-MAC.

a better method in terms of modal energy index. The reason for the results is that the MMAC matrix is computed using the new modal shape matrix $\bar{\Phi}$, which is composed by the modal vectors with large dynamical response.

The data in Table 5 show that IAGA-MMAC gets the smaller RMS and maximum of off-diagonal elements than the other two methods. A similar conclusion can be achieved from Figures 14 to 16. The result in Figure 17 shows that the IAGA-MMAC method has a faster evolution velocity and better fitness function value than AGA-MAC. The reason for the result is that the improved adaptive crossover and mutation factors enhance the searching efficiency and global optimization ability of the algorithm.

4.6. Discussion. The demonstration case is based on a numerically simulated wharf structure. The FE model of the wharf

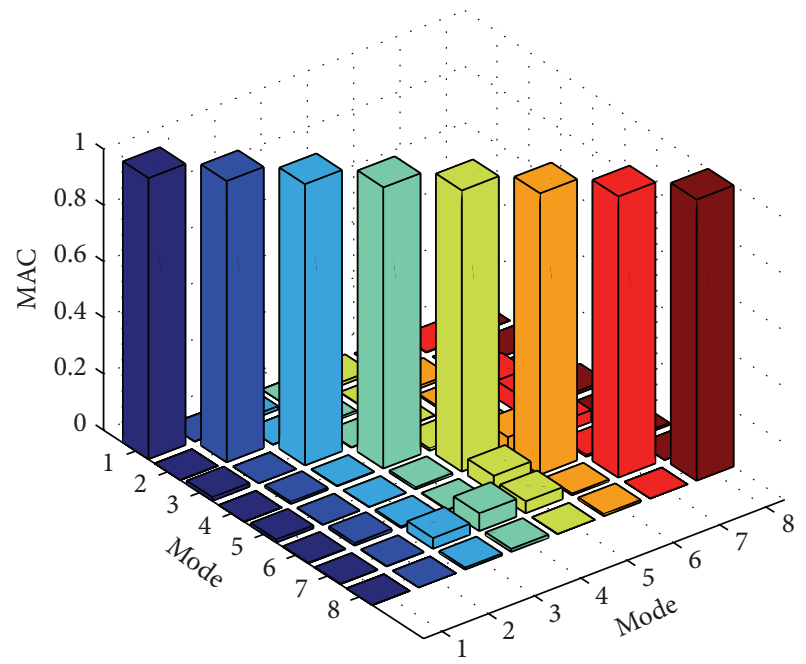

FIGURE 15: MAC matrix of IAGA-MAC.

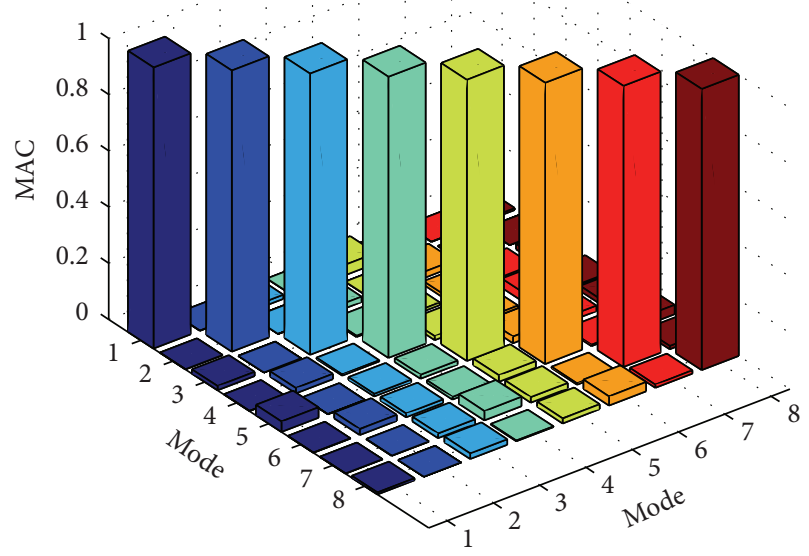

FIGURE 16: MMAC matrix of IAGA-MMAC.

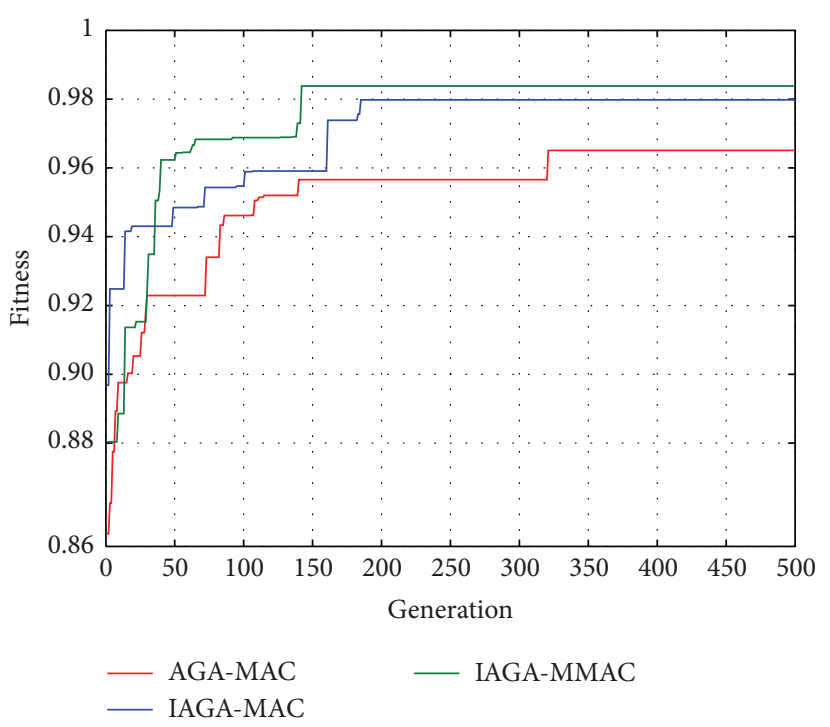

FIGURE 17: Evolution curve of three methods. 
structure is built in strict accordance with the actual structure. Therefore, the numerical simulation can verify the effectiveness of the IAGA-MMAC method to a certain extent. Of course, the numerical FE model maintains some limitations inevitably. Firstly, noise and other environmental factors, such as temperature and wind force, may impact the dynamic feature of the real wharf structure. However, these factors are not considered in the simulation experiment. Secondly, in the real structure, there are some locations that are not available to deploy the sensors. But these nodes may be selected as the optimal sensor locations in the numerical analysis.

\section{Conclusions and Future Work}

There are some shortcomings existing in the traditional minMAC method, such as a low modal energy and a large computational complexity. In order to solve these defects, a new method termed IAGA-MMAC is presented in this study. In the strategy, the traditional MAC is modified by constructing the new modal shape matrix to improve modal strain energy of the selected sensor locations. And then, IAGA, using the MMAC matrix as optimization objective function, is adopted to enhance the computation efficiency. With the case analysis, some conclusions are summarized as follows.

(1) The traditional MAC only concerns the correlation of the measured modal vectors. Therefore, it is necessary for the criterion to be modified to improve the modal strain energy of the selected locations. In the modified criterion, MPF is adopted to evaluate the dynamical feature of different modal orders firstly. And then, the new modal shape matrix $\bar{\Phi}$ is constructed by the modal orders which have big vibration response. Finally, the MMAC matrix is computed using the new modal shape matrix. As experiment results showed, MMAC can enhance the MSE of the selected locations.

(2) Due to the improved adaptive crossover and mutation factors, IAGA has a good searching ability and computation efficiency. Therefore, IAGA using the MMAC matrix as fitness function is presented in this study to achieve OSP. Experiment results show that IAGA-MMAC has a faster computational speed and a better optimization ability than AGA-MAC and IAGA-MAC.

The IAGA-MMAC method is proved to be an effective OSP method as the numerical analysis. However, there are still some works to do. An experiment of sensor placement on a real wharf structure will be conducted by our team in the further research.

\section{Conflict of Interests}

The authors declare that there is no conflict of interests regarding the publication of this paper.

\section{References}

[1] B. Bhattacharya, "Disaster mitigation of large infrastructure systems," in Proceedings of the International Symposium on Engineering under Uncertainty: Safety Assessment and Management (ISEUSAM '12), pp. 477-482, Bombay, India, January 2013.

[2] S. Jang, H. Jo, S. Cho et al., "Structural health monitoring of a cable-stayed bridge using smart sensor technology: deployment and evaluation," Smart Structures and Systems, vol. 6, no. 5-6, pp. 439-459, 2010.

[3] B. Li, D. Li, X. Zhao, and J. Ou, "Optimal sensor placement in health monitoring of suspension bridge," Science China Technological Sciences, vol. 55, no. 7, pp. 2039-2047, 2012.

[4] C. Boller, F. K. Chang, and Y. Fujino, Encyclopedia of Structural Health Monitoring, John Wiley \& Sons, New York, NY, USA, 2009.

[5] J.P. Ou and H. N. Li, "Structural health monitoring in mainland china: review and future trends," Structural Health Monitoring, vol. 9, no. 3, pp. 219-231, 2010.

[6] D. Balageas and C. P. Fritzen, Structural Health Monitoring, ISTE, London, UK, 2006.

[7] M. Salama, T. Rose, and J. Garba, "Optimal placement of excitations and sensors for verification of large dynamical systems," in Proceedings of the 28th Structures, Structural Dynamics, and Materials Conference, pp. 1024-1031, Pasadena, Calif, USA, January 1987.

[8] N. Debnath, A. Dutta, and S. K. Deb, "Placement of sensors in operational modal analysis for truss bridges," Mechanical Systems and Signal Processing, vol. 31, no. 8, pp. 196-216, 2012.

[9] D. C. Kammer, "Sensor placement for on-orbit modal identification and correlation of large space structures," Journal of Guidance, Control, and Dynamics, vol. 14, no. 2, pp. 251-259, 1991.

[10] M. Meo and G. Zumpano, "Optimal sensor placement on a large scale civil structure," International Society for Optics and Photonics, pp. 108-117, 2004.

[11] L. Qin and X. R. Zhang, "Successive sensor placement for modal paring based-on QR factorization," Journal of Vibration Measurement \& Diagnosis, vol. 27, no. 3, pp. 1-8, 2001.

[12] D.-S. Li, H.-N. Li, and C.-P. Fritzen, "A note on fast computation of effective independence through QR downdating for sensor placement," Mechanical Systems and Signal Processing, vol. 23, no. 4, pp. 1160-1168, 2009.

[13] C. Papadimitriou, J. L. Beck, and S.-K. Au, "Entropy-based optimal sensor location for structural model updating," Journal of Vibration and Control, vol. 6, no. 5, pp. 781-800, 2000.

[14] H. Wang, G. Pottie, K. Yao, and D. Estrin, "Entropy-based sensor selection heuristic for target localization," in Proceedings of the 3rd International Symposium on Information Processing in Sensor Networks, pp. 36-45, April 2004.

[15] T. G. Carne and C. R. Dohrmann, "A modal test design strategy for model correlation," in Proceedings of the 13th International Society for Optical Engineering Conference, pp. 927-933, New York, NY, USA, February 1995.

[16] X. Wang, J.-J. Ma, S. Wang, and D.-W. Bi, "Distributed particle swarm optimization and simulated annealing for energyefficient coverage in wireless sensor networks," Sensors, vol. 7, no. 5, pp. 628-648, 2007.

[17] S. Fidanova, P. Marinov, and E. Alba, "Ant algorithm for optimal sensor deployment," Computational Intelligence, vol. 399, no. 5, pp. 21-29, 2012. 
[18] N. Kukunuru, B. R. Thella, and R. L. Davuluri, "Sensor deployment using particle swarm optimization," International Journal of Engineering Science and Technology, vol. 10, no. 2, pp. 53955401, 2010.

[19] T.-H. Yi, H.-N. Li, and X.-D. Zhang, "A modified monkey algorithm for optimal sensor placement in structural health monitoring," Smart Materials and Structures, vol. 21, no. 10, Article ID 105033, pp. 1-9, 2012.

[20] W. Liu, W.-C. Gao, Y. Sun, and M.-J. Xu, "Optimal sensor placement for spatial lattice structure based on genetic algorithms," Journal of Sound and Vibration, vol. 317, no. 1-2, pp. 175-189, 2008.

[21] T.-H. Yi, H.-N. Li, and M. Gu, "Optimal sensor placement for health monitoring of high-rise structure based on genetic algorithm," Mathematical Problems in Engineering, vol. 2011, Article ID 395101, 12 pages, 2011.

[22] A. A. Javadi, R. Farmani, and T. P. Tan, "A hybrid intelligent genetic algorithm," Advanced Engineering Informatics, vol. 19, no. 4, pp. 255-262, 2005.

[23] T.-H. Yi and H.-N. Li, "Methodology developments in sensor placement for health monitoring of civil infrastructures," International Journal of Distributed Sensor Networks, vol. 2012, Article ID 612726, 11 pages, 2012.

[24] T.-H. Yi, H.-N. Li, and G.-X. Wang, "Optimal sensor placement for super high-rise building based on simplified finite element model," Chinese Journal of Computational Mechanics, vol. 25, no. 4, pp. 417-423, 2008.

[25] E. L. Wilson, Three-Dimensional Static and Dynamic Analysis of Structures, Computers \& Structures, California, Calif, USA, 2002.

[26] M. Srinivas and L. M. Patnaik, "Adaptive probabilities of crossover and mutation in genetic algorithms," IEEE Transactions on Systems, Man and Cybernetics, vol. 24, no. 4, pp. 656667, 1994.

[27] C. He, J. C. Xing, and J. L. Li, "A combined optimal sensor placement strategy for the structural health monitoring of bridge structures," International Journal of Distributed Sensor Networks, vol. 2013, Article ID 820694, 9 pages, 2013. 


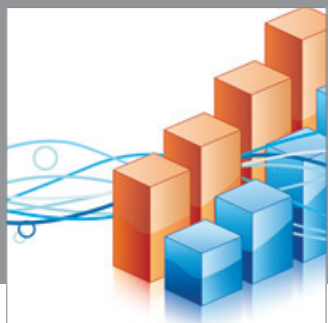

Advances in

Operations Research

mansans

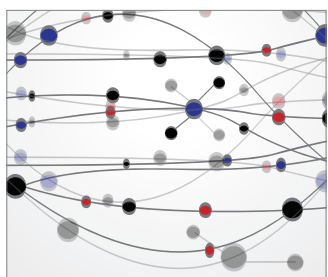

The Scientific World Journal
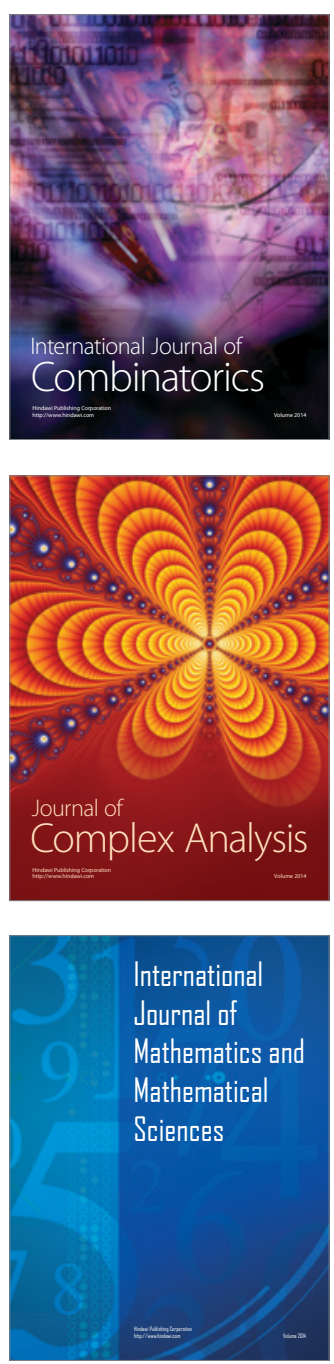
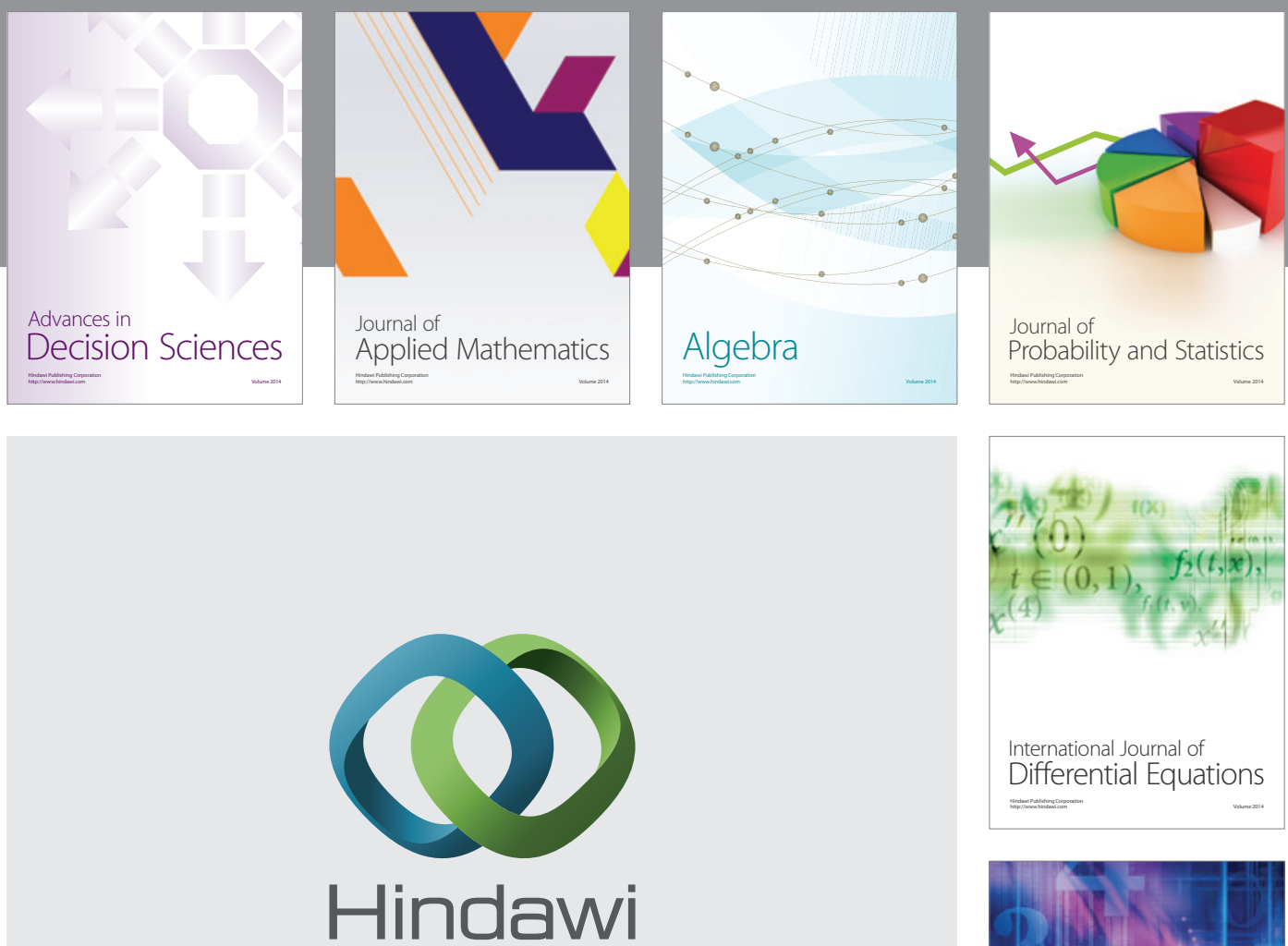

Submit your manuscripts at http://www.hindawi.com
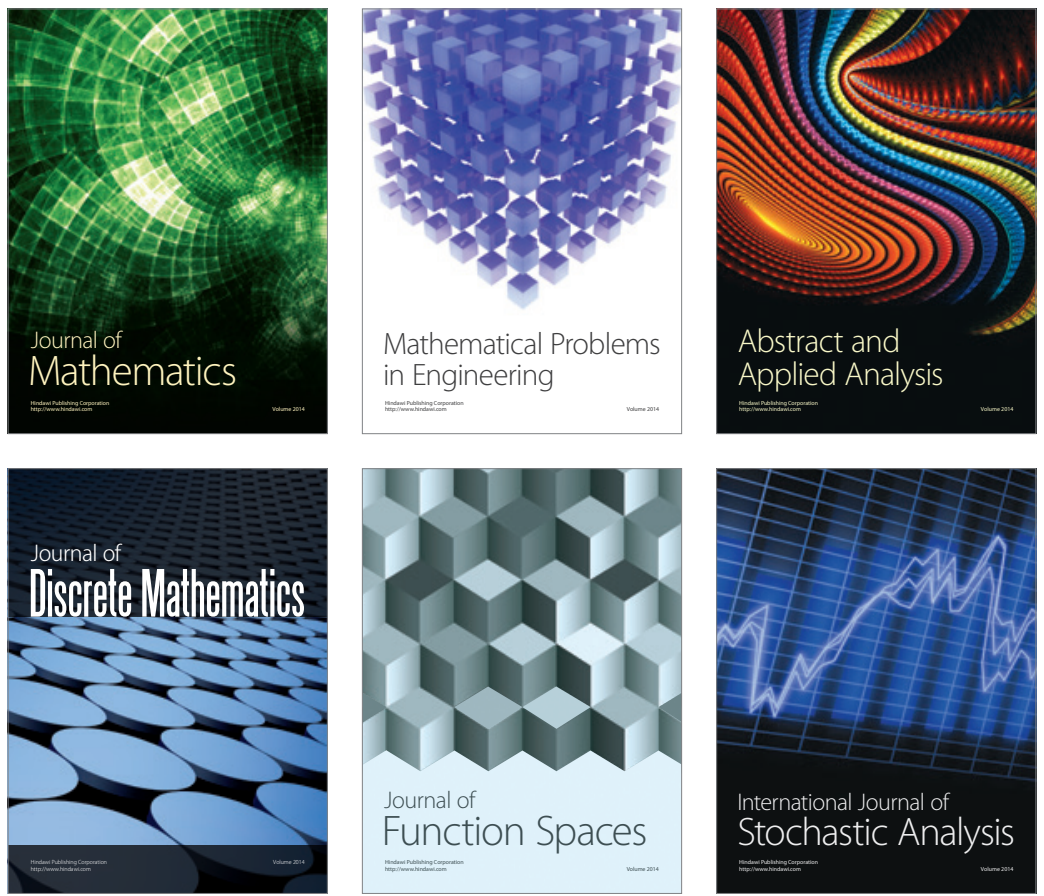

Journal of

Function Spaces

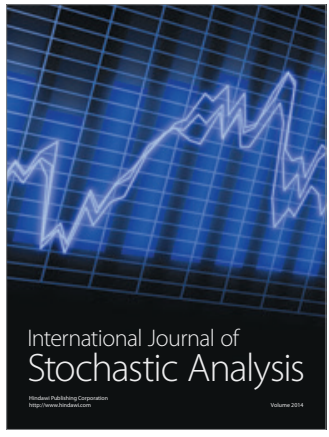

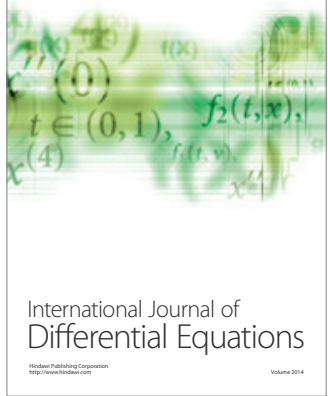
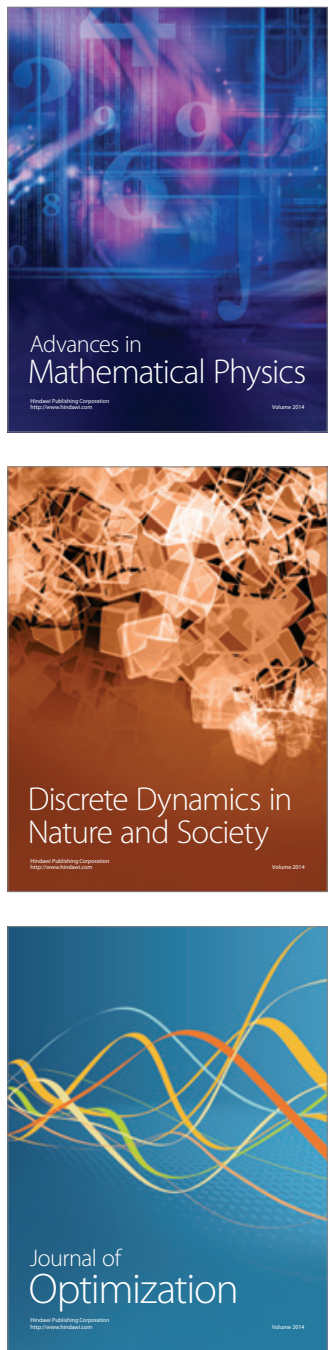